\title{
A THEORY OF CHEMICAL REACTION AND REACTIVITY
}

THE expression of a chemical reaction by an equation 1 which satisfactorily accounts for the numbers of the molecules taking part therein and the volumes of the reactants and resultants is too frequently accepted as being the last word on the subject. A somewhat sweeping statement perhaps, but I think it is justified when we consider how much is still left unexplained and how great is our ignorance of the fundamental phenomena of chemical change. We may select, as an instance, one of the simplest and most familiar of all reactions, namely, the combination of hydrogen and oxygen to form water, with its equation

$$
2 \mathrm{H}_{2}+\mathrm{O}_{2}=2 \mathrm{H}_{2} \mathrm{O} \text {. }
$$

It is remarkable how small has been the increase in our knowledge of this reaction since the classic work of Gay Lussac and Humboldt. It is true that we have far more accurate information as to the exact volumes and masses of the components. It is true that we have learned the amount of energy lost in the reaction, but, in spite of these and other advances in our knowledge, the smallness of that knowledge becomes apparent when we consider how much we do not know of this reaction. We do not know why hydrogen and oxygen atoms combine together at all. We do not know how they combine, and we do not know why they do not combine instantaneously when mixed, but 
require some external stimulus such as heat or an electric spark. I cannot but confess that these three questions to me present a fascinating problem, the solution of which would mean a great advance in our understanding of one of the most interesting of all natural phenomena, namely, chemical change.

In my previous lecture $I$ indicated how some light was thrown on this problem by absorption spectra observations, because in two different reactions, namely, the conversion of the aromatic aminoaldehydes and aminoketones into their salts, and the sulphonation of the phenols, the first stage of the reaction consists in the conversion of the aldehyde, ketone, or phenol into an intermediate stage which then undergoes the expected reaction. The observations offer the additional evidence that this intermediate stage consists of the original substance with a definite increment of energy, the amount of which can be measured by the difference between the frequencies of the absorption bands of the compound in the two energy states. The existence of this intermediate phase of the reactant, with an energy content higher than the normal, is not represented in the recognised equation of the reaction; and thus we learn that an entirely novel phenomenon is associated with, at any rate, the two reactions mentioned. In short, the reaction consists of two separate and distinct stages.

During recent years considerable attention has been paid to the energy relations of chemical reactions, and the modern theory is that every chemical process is preceded by this preliminary stage in which the reactants, chemically inactive at the start, are rendered chemically reactive by the supply of a definite increment of energy to each of the molecules. I do not propose in this lecture to discuss the work which has led to this important generalisation, for 


\section{Reaction and Reactivity}

I wish to confine myself to a discussion of the phenomena of reaction and reactivity from the point of view of absorption spectra. With these observations as our starting point, it seems possible to formulate a theory which correlates reaction and reactivity with absorption spectra and offers an explanation of both sides of the problem.

It will be remembered that in the previous lecture I gave no explanation of the phenomenon of absorption, my reason for this being that, as long as there is no understanding of the origin of the characteristic frequencies of molecules, it is useless to attempt to explain absorption spectra. The customary practice of accepting these characteristic frequencies as the gift of nature to molecules is in itself highly unsatisfactory. It is often loosely stated that the frequencies in the extreme ultra-violet are due to the electrons, the frequencies in the visible and near ultra-violet to the component atoms, and those in the infra-red to the molecules. This statement does not help us at all, nor does it carry us any further, since the absorptive power must be due to the existence of free periods of vibration in the molecule; and it is difficult on this basis to account for the undoubted increase in the energy content of the molecule when it vibrates in sympathy with radiant energy of the same frequency.

Again, it is a well established fact that when two atoms combine together energy is lost in the process. When, for instance, an atom of sodium combines with an atom of chlorine, energy escapes to the surroundings and the molecule of sodium chloride contains less energy than the free atoms of sodium and chlorine. Where does that energy come from? It surely means that the atoms when in combination possess less energy than when they exist in the free state. Accepting the assumption that the free atoms 
possess free periods of vibration it is not easy to understand how this enables those atoms to pass from a higher to a lower energy level. Further, we have yet to find an explanation of the fact, established by absorption spectra observations, that a molecule in passing from one phase to another changes its characteristic frequency.

These facts lead me to suggest that it is necessary to discard the theory of free periods of vibration as inadequate to explain well established phenomena. In my previous lecture I dealt with the integral relations between the various frequencies characteristic of a molecule, and I showed that their existence is an absolute necessity if the Planck hypothesis of energy quanta is sound. These relations, however, do not explain either the phenomenon of absorption or the Planck conception of quanta, yet they are observed facts, whilst the Planck quantum is only a hypothesis. For my own part, I believe that we shall approach more nearly to the truth if we postulate the exact converse of the commonly accepted theory. Whereas this theory postulates the existence of characteristic frequencies, and superimposed on that we have the Planck concept of energy quanta, I suggest that atoms and molecules are characterised by definite quantities of energy and that these determine the frequencies. In formulating this suggestion the observed facts of the integral relations between the absorption bands characteristic of a molecule are taken as the basis, and not the hypothesis of discontinuous radiation and emission as applied by Planck to the unexplained natural phenomenon of absorption bands.

The starting point of the new theory must obviously be the atom, since we have to account for the energy lost when two atoms combine together; and we, therefore, must state, as our principal assumption, that every atom is char- 


\section{Reaction and Reactivity}

acterised by a definite amount of energy which may be called the atomic quantum of energy. Every atom of the same element is characterised by the same atomic quantum, but the quanta of the atoms of different elements are different and characteristic of the elements. On this assumption an atom can only gain or lose energy in terms of its quantum, and it may be postulated that this quantum is associated with some physical change taking place within the atom, such, for example, as the shift of an electron from one stationary orbit to another.

In the second place, it may be assumed that this physical change within the atom takes a definite period of time which is the same for all atoms. Since the total energy absorbed is proportional to the frequency and the time, we may put

$$
\frac{\text { Atomic Quantum }}{\mathrm{T}}=\text { Frequency, }
$$

where $\mathrm{T}$ is a function of the time occupied by the physical change. It follows, from these two assumptions, that the atomic quantum endows the atom with the power of absorb. ing or radiating its quantum as radiant energy of a definite frequency, and the atom may be said to possess a characteristic frequency. These atomic quanta are very small and are of the order of $6 \times 10^{-16} \mathrm{erg}$. The frequency established by these quanta is of the order of $9 \times 10^{10}$, the corresponding wave-length being about $3200 \mu$.

Let us now consider the combination of two atoms together to form a binary molecule and let the characteristic quanta of these two atoms be $6 \times 10^{-16} \mathrm{erg}$ and $7 \times 10^{-18}$ erg, respectively. Accepting it as an observed fact that energy is evolved in this process, we may attribute the phenomenon to the loss by the two atoms of a certain number of their atomic quanta, that is to say, the total energy loss 
is shared in by the two atoms. In order to investigate the result of this it is necessary to have some basis to go on, and we may assume as a working hypothesis that the simplest possible sharing takes place, namely, that each atom contributes exactly half of the total energy loss. Two atoms, therefore, characterised by quanta equal to $6 \times 10^{-18}$ erg and $7 \times 10^{-18} \mathrm{erg}$, respectively, each lose the same amount of energy. The minimum amount of energy lost by each must be 7 quanta of the size $6 \times 10^{-18} \mathrm{erg}$ in the one case and 6 quanta of the size $7 \times 10^{-16}$ in the other case. The total energy loss, therefore, is twice the least common integral multiple of the two atomic quanta and is equal to $2 \times 6 \times 7 \times 10^{-16}=8.4 \times 10^{-15} \mathrm{erg}$. In order to resolve the resulting molecule back into its component atoms this quantity of energy must be absorbed by that molecule, and hence is characteristic of the molecule, since it is the quantity of energy required to decompose it. Similarly, if this molecule as a whole evolves energy, this must be a process in which its component atoms share equally, and hence the minimum quantity of energy which this molecule as a whole can absorb or radiate is $8.4 \times 10^{-15}$ erg. This quantity of energy may therefore be called the molecular quantum, and hence the molecular quantum must always be based on the least common integral multiple of the quanta of its component atoms. A molecule, therefore, will be characterised by a specific frequency established by its molecular quantum, and this frequency may be called the fundamental molecular frequency.

According to this view the chemical combination of atoms consists in the joint loss of equal amounts of energy by those atoms, and we may postpone the consideration of the affinity between them which causes them to enter into this process. The important point at the moment is that 


\section{Reaction and Reactivity}

the theory can be put to the test of experimental observation. It is evident in the first place, on this theory, that chemical reaction can only take place between atoms which are capable of losing energy, and in the second place, that in entering into combination, atoms do not lose their individuality as absorbers or emitters of energy, for they still will be able to absorb or radiate their characteristic atomic quanta. On examination with the spectroscope a molecule will exhibit its own fundamental frequency and also those frequencies characteristic of its atoms, and the least common multiple principle should obtain between these. As a matter of fact, these relations have been observed and they afford striking evidence in support of the theory now brought forward. ${ }^{1}$

An important proviso must be made in connection with the atomic quanta and their least common multiples, since it is not possible to speak of the least common multiple of incommensurable quantities. In dealing with atomic quanta and in equating two amounts of energy, each consisting of an integral number of quanta, the tacit assumption was made that the two quanta were commensurable. It must not be forgotten, however, that these atomic quanta will not be commensurable unless they are expressed on the same fundamental scale. In order that they can be expressed on the same scale the atomic quanta must themselves be integral multiples of a fundamental unit of energy. It is impossible here to enter into the arguments which justify the existence of this fundamental unit, but they may be summed up by saying that did it not exist it would be utterly impossible to conceive of any correlation between the frequencies and energy contents of substances, and in-

\footnotetext{
'Baly, Astrophys. Journ., 42, 1 (1915); Phil. Mag., 39, 565 (1920); Garrett, ibid. 31, 505 (1916); Baly and Garrett, ibid. 31, 512 (1916).
} 
deed the absorption of energy at one frequency and its radiation at another frequency would be impossible on the Planck theory. ${ }^{1}$

In dealing with the absorption by atoms of their atomic quanta of energy there is no need to assume that a single atom can only absorb one quantum at a time. It may absorb $1,2,3$, etc., quanta at the same instant, and consequently atoms will exhibit a series of frequencies $\mathrm{nA}$, where $\mathrm{A}$ is the frequency established by the atomic quanta and $\mathrm{n}=1,2,3$, etc. Since the probability of the atom doing this decreases with the number of quanta, the intensity of the absorbing frequencies $n A$ will decrease with increase in the value of $n$. This is actually observed, and molecules exhibit series of atomic frequencies with decreasing intensity. The significance of the least common multiple will perhaps be better understood if we call the molecular frequency the convergence frequency of the series of atomic frequencies.

$\mathrm{Up}$ to the present we have only dealt with the combination of two atoms to form a binary molecule, each atom being assumed to be characterised by one atomic quantum. There is no need, however, to restrict our first assumption in this way for it is to be expected that, at any rate in the atoms of some elements, there will exist more than one possibility of shift of electrons, and that there will be associated with such atoms two or more atomic quanta of different sizes. When atoms of this type enter into combination, exactly the same conditions apply as in the case of the binary molecule dealt with above, but a fresh phenomenon makes its appearance. This may be understood at once if we consider the simple case of two atoms, $A$ and $B$, combining together to form the molecule $\mathrm{AB}^{2}, \mathrm{~A}$ having two characteristic atomic quanta. Let the two atomic quanta

'Baly and Campbell, Phil. Mag., 41, 711 (1921). 


\section{Reaction and Reactivity}

of $A$ be represented by the numbers 2 and 3 respectively, and the atomic quantum of $\mathrm{B}$ by the number 5 . As before, the fundamental molecular quantum of $\mathrm{AB}^{2}$ will be based on the least common multiple of 2,3 , and 5 , that is to say, 30 , but we now have the possibility of two new quanta, namely, those of the atomic group $A B$. These two intramolecular quanta, as they may be called, will be the least common multiples of 2 and 5 , and of 3 and 5 , respectively, one being caused by the combination of one atom of $B$ by virtue of one atomic quantum of $A$, and the other by the combination of the second atom of $B$ by virtue of the second quantum of $\mathrm{A}$.

When such a compound is examined spectroscopically it will be found to exhibit three sets of frequencies, the fundamental molecular frequency, two intra-molecular frequencies, and three atomic frequencies. This type of absorption spectrum has been found in the case of water and sulphur dioxide, and the quantitative relations between the various frequencies conform to the theory with remarkable accuracy. ${ }^{1}$

There is no need to discuss in detail the relations which will obtain in more complex molecules, since, though possibly more complicated, the same principles will hold as in the simpler molecules. Two very interesting experimental confirmations of these relations may be mentioned. In the first place it is obvious that an intra-molecular quantum is solely characteristic of a particular group of atoms and can in no way be modified by the rest of the molecule. It follows, therefore, that all compounds containing such atomic groups as $\mathrm{OH}, \mathrm{NH}_{2}, \mathrm{SO}_{3} \mathrm{H}, \mathrm{CH}_{3}, \mathrm{C}_{6} \mathrm{H}_{5}$, etc., will be characterised by the intra-molecular quanta of these groups and that they will show the frequencies character-

\footnotetext{
${ }^{1}$ Baly and Garrett, Phil. Mag., 31, 512 (1916); Baly, ibid., 39, 565 (1920).
} 
istic of these groups. In my previous lecture I drew attention to the discovery by Coblentz of infra-red frequencies characteristic of these groups, these being shown by all compounds containing the groups. ${ }^{1}$

A second interesting combination is afforded by naphthalene which exhibits an intra-molecular frequency of 1.4136 $\times 10^{13}$ and this must be characteristic of an atomic grouping within the naphthalene molecule. The two chief atomic groups in this molecule are the benzene ring and the olefine grouping. The frequency $1.4136 \times 10^{13}$ should be the fundamental molecular frequency of either benzene or the olefines, and, whilst no such frequency is found in the absorption spectrum of benzene, the characteristic frequency of the olefines is found from Coblentz's measurements to be $1.415 \times 10^{13}$.

There remains one further deduction which follows from this theory and leads to an important result from the point of view of the energy changes of a molecule. The fundamental molecular quantum is by definition equal to an integral number of atomic quanta characteristic of each of its component atoms, and is also equal to an integral number of the intra-molecular quanta characteristic of the groups of atoms forming part of the molecule. It follows, therefore, that a molecule can gain or emit one molecular quantum at any of those three frequencies. As will be seen later

${ }^{1}$ Pub. Carnegie Inst., Washington, No, 35 (1905). Time will not permit of the discussion of the principle of the combination of the various frequencies due to the simultaneous absorption of quanta of different sizes. In these combinations is found the explanation of the breadth and structure of absorption bands as actually observed. A full account of these will be found in the papers already referred to. These combined frequencies are of great importance as they enable us to determine the frequencies in the long wave infra-red where direct measurements bave not yet been made. 


\section{Reaction and Reactivity}

this deduction is of singular importance in connection with the energy changes involved in a chemical reaction.

In the foregoing I have dealt only with the quantitative relations which follow from the original assumption that every elementary atom is characterised by one or more quanta of energy, the whole being based on an observed fact, namely, that there exists some force of attraction which causes atoms to come together and jointly to lose energy. It is not difficult to find an explanation of this affinity in the electro-magnetic force fields of the atoms as has been pointed out by Humphreys, Sir J. J. Thomson, and others, but the explanation lacked a quantitative basis. On the other hand it seems probable that the theory of atomic and molecular quanta is able to supply the necessary quantitative foundation. The electromagnetic force fields of atoms were first dealt with by Humphreys, ${ }^{1}$ who showed that they are capable of giving a quantitative explanation of the Zeeman effect and also of the pressure-shift of spectrum lines. He deduced the fact that two atoms will attract one another when they approach in such a way that the direction of their electronic motions is the same, and will repel one another when their electronic motions are in opposite directions. Each atom, therefore, possesses two faces, and when one pair of faces comes together they attract one another, and when the other pair comes together they repel one another. In other words an atom forms the centre of an electro magnetic field of force, the opposite poles of which are localised in two opposite faces of the atoms.

Let it be supposed that two atoms of different elements are brought together in such a way that their mutually attracting faces come together. They will at once tend to form an addition complex, losing energy in the manner

${ }^{1}$ Astrophys. Journ., 33, 233 (1906). 
described above. The two atoms radiate equal amounts of energy as a whole number of elementary quanta, whereby the resulting molecule becomes endowed with a quantum based on the least common multiple of the atomic quanta. This molecule is now rendered a stable entity and can only be resolved into its atoms by absorbing an amount of energy equal to that lost in its formation.

As will be noticed, however, in this suggestion that the reactivity of atoms for one another is due to the attraction of their respective force fields and that their combination consists in their joint loss of equal amounts of energy, no account has been taken of the other faces of these combining atoms. Whereas the combination of the atoms produces a molecule characterised by a specific energy quantum, it is not possible to consider that the force fields due to the external atomic faces can exist without influence on one another. These external force lines must condense to form an external molecular force field and in this process energy must be radiated.

It was shown previously that a freshly synthesised molecule is characterised by a definite molecular quantum, and, therefore, when a freshly synthesised molecule loses energy as a whole it must do so in terms of its molecular quantum. It thus follows that, when the external force fields of the component atoms of a freshly synthesised molecule condense together to form the molecular force field, the system must lose a definite number of molecular quanta. Clearly the molecule itself will not suffer any loss of individuality as far as its characteristic quanta and frequencies are concerned. None of the deductions from the conception of elementary and molecular quanta made previously will be contradicted, and the only change accompanying the formation of the molecular force field will be the endowment 


\section{Reaction and Reactivity}

of the system with an additional quantum and frequency which are exact multiples of the fundamental molecular quantum and frequency, respectively. Let it be supposed that in the formation of its molecular force field a given molecule loses one molecular quantum. If the freshly synthesised molecule were allowed to absorb one molecular quantum, it would beçome endowed with certain properties. If, now, it is required to bring the molecule with its molecular force field established by the loss of one quantum into this physical state, it will be necessary to supply it with energy equal to two fundamental molecular quanta. There can be no reason against the molecule and its force field absorbing both these quanta simultaneously, and, therefore, it may be concluded that the system of molecule and force field becomes endowed with a new and additional quantum and frequency which are exactly twice the fundamental quantum and frequency. Similarly, it follows that if the force field condensation proceeds to the extent defined by the loss of two molecular quanta, the molecule and its force field will be endowed with a new and additional quantum and frequency which are exactly three times the fundamental quantum and frequency. Generally, if the fundamental quantum and frequency of a freshly synthesised molecule be denoted by $\mathrm{M}$ and $V$, and if in the formation of the force field $\mathrm{x}$ quanta are evolved, the system will be characterised by an additional quantum and frequency, namely, $M(x+1)$ and $V(x+1)$, respectively. Since the external atomic fields are bound to undergo a certain amount of condensation, it is evident that the molecule must exist in one of a number of possible phases, each molecular phase being defined by the number of molecular quanta lost in the force field condensation, and characterised by a quantum and a frequency which are exact multiples of the funda- 
mental quantum and frequency, respectively. Obviously, therefore, the difference in energy content between any two molecular phases will be one fundamental molecular quantum. It may be pointed out that the integral relationships between the frequencies and the existence of phases differing in energy content by one molecular quantum now deduced were also arrived at from absorption spectra observations as described in my previous lecture. The present theory, therefore, finds remarkable support in the experimental observations of absorption spectra.

In formulating our theory the initial assumption was made that the chemical reactivity of atoms is due to the attraction exerted by their electromagnetic fields. Similarly, the reactivity of molecules will be a function of their force fields, and it follows that the reactivity of a molecule will depend on the phase in which it exists. In other words, each phase will be characterised by its own reactivity, a conclusion that has also been independently arrived at from absorption spectra observations. Before attempting to apply this theory of molecular phases to the phenomena of chemical reaction and reactivity we may restate the whole theory and the deductions which have been made from it. The theory rests on three initial assumptions which are as follows :

1. Every elementary atom is characterised by one or more quanta of energy which are integral multiples of a fundamental unit of energy.

2. Atoms can only gain or lose energy in terms of their atomic quanta, and the process of emission or absorption of one quantum by an atom occupies a definite time which is the same for all atoms and may be equated to the Planck constant, $6.56 \times 10^{-27}$. 


\section{Reaction and Reactivity}

3. When atoms combine together the total energy loss is shared equally between them.

It follows, from these assumptions, that the quantum of energy characteristic of a molecule is based on the least common integral multiple of the quanta of its component atoms. Except in the case of binary molecules there will exist intra-molecular quanta, characteristic of groups of atoms within the molecule. The relations between the frequencies established by all these quanta have been directly observed experimentally.

The affinity or reactivity of atoms is attributed to their electromagnetic force fields, and, after atoms have combined together, their external force fields undergo condensation with the formation of an external molecular force field. This condensation takes place with the evolution of molecular quanta, and consequently a freshly synthesised molecule must pass into one of a number of possible phases, depending on the number of such quanta that are radiated. Each phase is characterised by its phase quantum and frequency, in addition to its molecular and atomic quanta and frequencies, by its specific reactivity, and by its energy content-any two consecutive phases differing in energy content by one molecular quantum. We thus arrive at conclusions which are identical with those drawn from absorption spectra observations.

In applying this theory to the phenomena of chemical reactivity and reaction we may first consider the case of elementary atoms, since it is necessary to discuss the probable nature of their force fields to which their reactivity is due. The simplest possible case will be that of an atom the electrons of which are rotating in orbits which lie in one plane. This will result in the establishment of an electromagnetic force field with its two poles equal and opposite. In this 
case it is evident that condensation must occur between the force lines of each pole with the evolution of a large number of atomic quanta and the formation of a highly condensed external force field. Such an atom will, therefore, exist in a phase of abnormally low energy content and will possess no measurable reactivity. It may be suggested that the atoms of the rare gases, helium, neon, argon, krypton, and xenon, are of this type, and that the non-reactivity of these atoms is due to their force fields being completely closed. The present theory does not permit of there existing a condition of a zero valency, since it is impossible to conceive of an atom with no power of losing energy. If, however, the non-reactivity of these gases is due to the abnormal condition of their force fields, it follows that they should become endowed with reactivity if the closed force fields were opened by the supply of energy. The realisation of this would entail a large amount of energy being given to the atom, such as only could be supplied at the frequency characteristic of the highly condensed phase. This frequency must lie in the very extreme ultra-violet region, and radiation of that frequency will be absorbed by air, quartz, and probably by all known substances. Special means would, therefore, be necessary to supply this energy and the most likely condition would seem to be the electric discharge in vacuo. It is, therefore, of more than passing interest that so many observers have recorded the "disappearance" of the rare gases when an electric discharge is passed through them under reduced pressures. It certainly does not seem to be beyond the bounds of possibility that, under the influence of the electric discharge which is known to radiate energy of very short wave-length, the rare gases absorb this energy at their phase frequencies and are converted into 


\section{Reaction and Reactivity}

reactive phases with the result that they combine with the metal of the electrodes.

On the other hand, if there exist electronic orbits which do not all lie in the same plane, an unsymmetrical force field will result, and condensation to give the highly condensed and non-reactive external field will not be possible. The two poles will no longer be equal and opposite, and the strength of one or other will predominate. The atoms will tend to lose the maximum possible amount of energy, either by themselves or by combining with one another to form molecules. The normal state will be one in which the free particles are in radiant equilibrium with their surroundings. I believe that it will be possible, when the electronic orbits are known, to derive the various types of molecules with which the chemist is familiar, namely, (1) the nonreactive diatonic molecule such as $\mathrm{H}_{2}, \mathrm{~N}_{2}$, etc., (2) the highly reactive monatomic molecule such as $\mathrm{Na}, \mathrm{K}$, etc., (3) the highly reactive diatomic molecule such as $F_{2}$, (4) the non-reactive polyatomic molecule such as that of carbon.

In the case of an incompletely closed external force field one pole will predominate and this will determine the nature of the reactivity of that element, whether it is electropositive or electronegative in type. Again, according to the theory of atomic quanta, an atom which is characterised by one quantum can only form a saturated molecule by combining with another atom of the same type. Such an atom will be univalent, whilst an atom characterised by two atomic quanta will have two powers of combining, that is to say it will be bivalent, and so on. The valency of an atom, therefore, would seem to be a measure of the number of atomic quanta characteristic of that atom.

When an atom is characterised by two or more atomic quanta, which must naturally be of different sizes, the 


\section{2}

\section{Photochemistry}

largest of these will be concerned first in any reaction, for it is safe to assume that a system will tend to fall to the lowest energy content possible under the conditions prevailing. In the case of such a molecule as $\mathrm{FeCl}_{3}$ the energy lost in the three successive combinations of the iron atom with the chlorine atom will progressively decrease. The energy lost when the first atom of chlorine combines will be the least common multiple of the largest iron quantum and the quantum of chlorine. When the second atom combines the energy lost will be the least common multiple of the second iron quantum and the chlorine quantum, whilst in the third combination the smallest iron quantum will function. This is well known to be the case from thermochemical measurements. Following on this it may be suggested that the position of a metal in the electropositive series is determined by the numerical size of its largest characteristic atomic quantum.

When we come to consider the phases in which molecules exist, it by no means follows from the general theory that all the molecules of a given substance must exist in the same phase. The observations of absorption spectra described in the previous lecture, whatever interpretation be put on them, show very conclusively that some form of equilibrium exists which is changed by varying temperature and solvent. These equilibria, according to the present theory, exist between two or more phases of the given molecules, and in one or two cases it has been found possible to determine the relative amounts of the component phases. Thus chlorine at $0^{\circ}$ and $760 \mathrm{~mm}$. exists as an equilibrium mixture of two phases with absorption bands having centres at 3380.8 and 926.73 Angstroms, respectively, whilst the relative numbers of molecules in each phase are 10 and $3496 .^{1}$

'Baly and Morton, J. Phys. Chem., 28, 659 (1924). 


\section{Reaction and Reactivity}

In the case of solid substances the possibility arises of the metastable existence of equilibria which are different from the normal phase equilibrium. This phenomenon would be of particular interest in the case of the solid elements, since it would offer an explanation of allotropy. Smits has independently put forward the same theory in that he considers that allotropic modifications of an element are different equilibrium mixtures of a limited number of different molecular species of that element. In the case of sulphur Smits interprets the various allotropes as equilibrium mixtures of the four molecular species $S_{\lambda}, S_{\mu}, S_{x}$, and $S_{\phi}$. On the present theory the molecular species of Smits is a definite phase of the sulphur molecule; and we possess the added advantage of a more accurate knowledge of the criteria of the phases, for we know the energy content and the characteristic frequency of each phase. It becomes possible, therefore, to examine the equilibria existing in the various allotropes of sulphur by absorption spectrum methods, and by comparison with the four varieties to determine directly the components present. Some preliminary observations of these absorption spectra have given definite evidence of the truth of this interpretation of allotropy of sulphur, since the frequencies characteristic of the four varieties are also characteristic of the allotropic modifications. The problem of sulphur, however, is not so simple as it appears at first sight, and the investigation is being continued.

The molecular phase hypothesis may now be applied to the phenomenon of chemical reaction. Amongst the deductions from this hypothesis I mentioned that each phase of a given molecule is characterised by its own chemical reactivity, and hence for any given reaction a molecule must be brought into the right phase. If in the normal phase equi- 
librium there are present no molecules in the appropriate phase, the substance will not react. Thus, in the instance quoted at the commencement of this lecture, namely, the combination of hydrogen and oxygen, there are present no molecules in the right phase and consequently no reaction takes place when the two gases are mixed.

In general, pure substances do not react with one another because no molecules in the appropriate phase are present. The well-known catalytic effect of certain impurities in pro. moting reaction will be discussed later. In actual fact, the molecules of pure substances exist in phases of lower energy content than the phase necessary for the reaction required. As a specific instance for detailed consideration we may select the reaction between hydrogen and chlorine which does not take place when the gases are mixed, the two phases known to be present in chlorine having no reactivity towards hydrogen. The reactive phase of chlorine has a larger energy content and by the supply of energy to the chlorine some of the molecules are converted into the reac. tive phase and the reaction commences. It is known that the two phases of chlorine absorb light of the wave-lengths 3380.8 and 926.73 Angstroms, and consequently rays of either wave-length should activate the chlorine molecules and induce the reaction with hydrogen to take place. IIlumination of chlorine by the light of the shorter wavelength is not practicable in air, but it is well known that light of the longer wave-length causes the union of hydrogen and chlorine to take place.

Owing to the integral relationships which exist between the phase quanta and the fundamental quantum it should theoretically be possible to cause the necessary phase change in chlorine by exposing it to radiation of the same frequency as the infra-red fundamental frequency, which is 


\section{Reaction and Reactivity}

$1.64325 \times 10^{13} \quad\left({ }_{x}=18.2565_{\mu}\right)$, since the phase change requires a whole number of the fundamental quanta. It must be remembered, if the phase change requires a large number of the fundamental quanta, that difficulties may arise. The probability against a single molecule absorbing a number of quanta increases with that number, since by the absorption of one quantum the molecule becomes a radiating system and therefore may lose that quantum before it absorbs the second. In the case of chlorine, however, the activation by infra-red radiation does seem to be possible, since the combination with hydrogen takes place when the mixed gases are heated.

The theory thus leads to the conclusion that in the case of all substances it is necessary to supply energy to the molecules in order to bring them into their reactive phases. Further, the amount of energy required for the phase change of any molecule depends on the energy contents of the initial and reactive phases and must always be exactly equal to a whole number of the fundamental molecular quantum. The molecular quantum of chlorine calculated from refractivity measurements is $1.078 \times 10^{-13} \mathrm{erg}$ per molecule, which is 1613 calories per gram molecule. The amount of energy required to activate a single molecule of chlorine must therefore be either $1.078 \times 10^{-13} \mathrm{erg}$ or some multiple of this.

The most important conclusion we have arrived at is that every chemical reaction must be preceded by a preliminary process in which the molecules of the reactants are activated. We see, therefore, that there must be at any rate two stages in every reaction, namely, activation and reaction proper. As a matter of fact, there is a third stage, as may readily be seen if we consider the reverse reaction, namely, the photochemical conversion of hydrogen chloride 
into hydrogen and chlorine. The first stage in this reaction will be the absorption of energy by the hydrogen chloride molecules whereby they are changed from their normal non-reactive phases to their reactive phase. It follows that the reaction proper takes place between the reactive phases of the components from whichever side we start. In any reaction, therefore, there must be three stages, namely:

1. Absorption of energy to change the non-reactive phases into the reactive phase.

2 . The reaction proper in which molecules of the products are formed in their reactive phases.

3. The loss of energy by the reactive phases of the products to give their normal and non-reactive phases.

It may be noted that the observed heat of any reaction, that is to say, the energy change that can be measured by means of a calorimeter, is the difference between the energy absorbed in the first stage and the energy radiated in the third stage. A reaction is endothermic or exothermic according to whether the first quantity of energy is greater or less than the second.

As described in the previous lecture, absorption spectra observations have definitely established the formation of the intermediate activated phase with larger energy content in two different reactions, namely, the conversion of certain organic bases into their salts and the sulphonation of certain phenolic compounds. These results are in exact agreement with the deductions made from the theory.

An important conclusion has been derived from the theory of the activation of molecules by a definite increment of energy as the preliminary stage of all reaction. If every molecule when activated by the definite increment of energy undergoes reaction, it follows that the number of molecules 


\section{Reaction and Reactivity}

reacting must be given by the total energy supplied divided by the definite increment. In the case of the photochemical reaction between hydrogen and chlorine, in which the chlorine is activated by light of its phase frequency in the ultraviolet, each chlorine molecule absorbs the phase quantum which is $5.821 \times 10^{-12} \mathrm{erg}$. If the mixed gases absorb $\mathrm{x}$ ergs, then the number of chlorine molecules which react should be $x / 5.821 \times 10^{-12}$ and the number of hydrogen chloride molecules formed should be $2 \mathrm{x} / 5.821 \times 10^{-12}$. This is known as Einstein's law of photochemical equiva. lence.

As a matter of experimental fact it has been found that this law does not hold except in very few cases, the number of molecules reacting being enormously greater than one for every quantum absorbed. In some reactions as many as 10,000,000 molecules react for every quantum absorbed. This divergence from Einstein's law has been considered by many as a very serious criticism of any theory of chemical reaction based on the Planck energy quantum theory, but it would seem very probable on the present theory that the number of reactant molecules must greatly exceed the number of quanta absorbed in any exothermic reaction. The Einstein law is based on the first stage of the reaction only, and takes no account of the energy radiated during the third stage. This energy is radiated at frequencies characteristic of the resultants, and, since these contain the same elements as the reactants, some of this energy will be reabsorbed by the reactants with the result that more will be activated than the Einstein law requires. The proportion of the radiated energy that is re-absorbed will depend on the density of the radiation and on the density of the reactants. It has been experimentally proved that the divergence from Einstein's law does increase with increase 


\section{8}

\section{Photochemistry}

both in the density of the radiation and in the density of the reactant that is being activated. I believe that this affords the strongest possible evidence in support of the present theory. Further, it would follow that in an endothermic photochemical reaction the Einstein law should hold, since the energy available for re-absorption by the reactants is small. In the photochemical conversion of oxygen into ozone, an endothermic reaction, the law has been proved to hold good. ${ }^{1}$

This theory also offers an explanation of the variation in absorption spectra frequently observed with change in solvent, the variation being due to new phase equilibria being established. Since the absorption bands exhibited by one and the same compound under various conditions of temperature and solvent are characteristic of the different phases present, the integral relations between the frequencies should be in accordance with the present theory, and it has already been shown that they are exactly those required by the theory. No attention has as yet been directed to the mechanism whereby a solvent can supply energy to a substance and change the phase equilibrium. Let it be supposed that when a substance dissolves it forms an addition complex with the solvent molecules. There are two ways in which a complex of two or more molecules can be stabilised. The formation of the complex may be accompanied by the radiation of energy to the surroundings, in which case the complex cannot be dissociated unless that energy is restored to the system. On the other hand, a complex may be formed by the transference of one or more energy quanta from one component to the other, in which case the complex will not dissociate unless the amount of energy, equal to

${ }^{1}$ For a complete discussion of these phenomena see Phil. Mag., 40, 23 (1920). 


\section{Reaction and Reactivity}

that transferred, is supplied to it. Absorption spectra measurements should show in either type of complex changes of phase compared with those present in the components. When a complex of the first type is formed both components lose energy, with the result that the phases present in the complex will exhibit a larger characteristic frequency than those shown by the free components. In the formation of the second type of complex one component gains energy at the expense of the other, and consequently the absorption spectra of the two will change in opposite senses. Any phase change on the part of one component will be accompanied by a corresponding phase change on the part of the other in the opposite direction. In this case the frequency characteristic of one component in the free state will shift towards the red, whilst the frequency characteristic of the other will shift towards the ultra-violet.

Some observations have been made of the absorption spectra of two liquid substances, the characteristic frequencies of which lie in the visible or ultra-violet, and of mixtures of the two, and the changes noted on mixing are exactly those expected on this hypothesis. The different phase of a given substance present in solution in different solvents may therefore be understood. It is very possible also that the phenomena of catalysis may be explained in the same way, the catalyst having the power of forming complexes with the catalyte, in which the catalyte is converted into the reactive phase required for the particular reaction that is accelerated.

The influence of the solvent molecules in changing the phase of a reactant need not be restricted to the cases in which a solution is actually formed, since the same conditions may apply to any mixture of substances. The re- 
markable influence of water vapour in catalysing chemical reaction may well be due to the fact that it maintains some of the molecules of the reactant in the reactive phase. Since these form one component of an equilibrium mixture, the presence of very few will enable the reaction to take place. It is a necessary consequence that if every trace of water be removed, the activated phase of the reactant molecules will disappear from the equilibrium and the substance will become non-reactive. The loss of all power of reaction after intensive drying is a phenomenon made familiar to us all by the classic investigations of $H$. B. Baker, and in all probability the explanation is to be found in the fact that the water vapour when present converts some of the molecules into the reactive state. Here again the explanation can be put to the test of experiment, since, if it be correct, there will be definite evidence of a change in phase towards a condition of lower energy content on drying. Since the majority of the substances investigated by Baker only exhibit absorption bands in the extreme ultra-violet, the ordinary methods of absorption spectra are not suited for comparing the moist and dry substances. As has been previously stated the refractivity of a substance is connected with its characteristic frequencies in the extreme ultra-violet, and the refractivity will decrease if a phase change takes place accompanied by a loss of energy. It follows that the refractivity will decrease with intensive drying if the explanation now brought forward is correct. An investigation of the refractivities of moist and intensively dried gases is now being made in Liverpool, but the results although promising are not as yet ready for publication.

On the other hand, a very interesting positive result has been obtained with liquid benzene which shows a great decrease in refractivity on intensive drying. In the case of 
liquids Baker has found a marked increase in the boiling point after intensive drying, and this is an obvious result if the drying causes the molecules to pass into a phase or phases of smaller energy content. Since the vapour obtained by boiling the two samples of benzene, ordinary and dry, is normal, a greater amount of energy must be supplied to the dried liquid to cause it to boil and consequently the boiling point will be higher. The refractivity of benzene can be expressed in terms of absorption bands in the ultraviolet, the frequencies of which are integral multiples of the frequency $1.5071 \times 10^{13}$. This, therefore, may be taken as the fundamental frequency of benzene and $1.5071 \times$ $10^{13} \times 6.56 \times 10^{-27}=9.88666 \times 10^{-14}$ as the fundamental molecular quantum. If, therefore, every benzene molecule loses one molecular quantum on drying, the total amount of energy lost by one gram molecule will be $9.88666 \times 10^{-14} \times 6.22 \times 10^{28} \div 4.191 \times 10^{7}=1467$ calories, and this extra amount of energy must be supplied to one gram molecule of the intensively dried benzene in order to cause it to boil. Now the specific heat of benzene at $94^{\circ}$ is 0.481 calorie per gram or $0.481 \times 78=37.518$ calories per gram molecule and it follows that the energy increment of 1467 calories will raise the boiling point by $1467 \div 37.518=39.1^{\circ}$. Since the boiling point of ordinary benzene is $80.1^{\circ}$ the boiling point of the intensively dried benzene should be $80.1+39.1=119.2^{\circ}$. In his original paper Baker stated that the boiling point is $118^{\circ}$, but more recently he has found the boiling point to be $119^{\circ}$ which is strikingly close to the calculated value. ${ }^{1}$

In connection with the probability that the effect of intensive drying is to establish a new phase equilibrium of lower energy content, a brief reference may be made to

\footnotetext{
${ }^{1}$ Private communication.
} 


\section{2}

\section{Photochemistry}

some experiments on the thermal decomposition of ammonia. ${ }^{1}$ It was found that ordinary ammonia dried by quick-lime requires much less heat energy to decompose it than does ammonia obtained by explosive evaporation of the liquefied gas. It was further found that traces of water vapour added to the gas dried by quick-lime materially reduces the amount of energy required to decompose it. So far as the effect of water vapour is concerned the results support the explanation given of the effect of intensive drying. As regards the smaller reactivity of the gas obtained by explosive evaporation of the liquefied gas, this is quite analogous to the cases of pyridine, piperidine, etc., the vapours of which exist in phases of higher energy content than those present in the liquid. If the same is true of ammonia, the gas obtained by explosive boiling of the liquid will contain phases of lower energy content than the normal gas, with the result that more energy will be required to decompose it.

Time does not permit me to quote many other experimental observations which support the theory, and, indeed, repetition tends to weariness. In spite of this there is one question which hardly can be avoided, namely, where does the ionic theory find a place in our theory? The answer to this question is a simple one for the phenomenon of ionisation may be a property characteristic of a specific molecular phase of an electrolyte. The molecular phase hypothesis attributes the reactivity of an electrolyte in solution not to the ions but to the molecular phase which forms those ions in the given solvent. The reactivity of an electrolyte will be the same in two solvents which maintain the same equilibrium of reactive phase, even if the amount of ionisation is different in the two solvents. This

\footnotetext{
${ }^{2}$ Baly and Duncan, Trans. Chem. Soc, 121, 1008 (1922).
} 


\section{Reaction and Reactivity}

view has the advantage of correlating facts which on the ionic theory seem almost irreconcilable. For instance I need only mention the instantaneous and quantitative precipitation of copper chloride when benzene solutions of copper oleate and hydrogen chloride are brought together, the ionisation in the two solutions being negligible. ${ }^{1}$ It must be admitted that the experimental proof is difficult, since in general the absorption bands of electrolytes are situated in the extreme ultra-violet and the refractivities of mixtures are not yet understood. On the other hand, we have indisputable evidence of the reactivity of the nonionised molecules present in equilibrium with their ions. Perhaps also some results obtained in the absorption spectra measurements of violuric acid and its potassium salt in aqueous solution are of interest. It has always been believed that the colour of these solutions is due to the violuric ion, the colour being caused by a definite absorption band. ${ }^{2}$ The absorptive power at the central frequency of that absorption band is proportional to the ionisation, which is exactly what would have been expected on the ionic theory or the molecular phase theory. If, however, a large amount of potassium nitrate be added to a solution of potassium violurate the absorptive power is not altered. This proves that the absorptive power is due to the reactive phase and not the violuric ion, since the ionisation of the potassium violurate is materially decreased by the addition of the potassium nitrate. Corroborative evidence may also be found in the observation by Garrison ${ }^{3}$ that the conductivity of a suspension of copper coated with a thin layer of cuprous oxide is increased by light.

\footnotetext{
${ }^{1}$ Kahlenberg, Trans. Faraday Soc., 1, 42 (1905).

'Donnan and Schneider, Trans. Chem. Soc, 95, 956 (1909).

"J. Phys. Chem., 28, 279 (1924).
} 


\section{Photochemistry}

In conclusion, may I say that in putting forward this theory of chemical reaction and reactivity $I$ am fully aware that there is much experimental work yet to be carried out before it will be possible to say that it has fully stood the test of experiment? So far as the observations have been carried I have some confidence in saying that they uniformly support the theory. The deductions as to the existence of molecular phases and their properties made from absorption spectra observations are identical with those made from the theory. The support, however, given to the theory is found, not only in absorption spectra observations, but also in many other lines of work. 